\title{
The first meiosis of resynthesized Brassica napus, a genome blender
}

\author{
E. Szadkowski ${ }^{1}$, F. Eber ${ }^{1}$, V. Huteau ${ }^{1}$, M. Lodé ${ }^{1}$, C. Huneau ${ }^{2}$, H. Belcram ${ }^{2}$, O. Coriton ${ }^{1}$, M. J. Manzanares- \\ Dauleux $^{1}$, R. Delourme ${ }^{1}$, G. J. King ${ }^{3}$, B. Chalhoub ${ }^{2}$, E. Jenczewski ${ }^{4}$ and A-M. Chèvre ${ }^{1}$ \\ ${ }^{1}$ INRA, UMR118 APBV, BP35327, F-35653 Le Rheu Cedex, France; ${ }^{2}$ Organization and Evolution of Plant Genomes, URGV, UMR INRA 1165-CNRS \\ 8114-UEVE, F-91057 Evry, France; ${ }^{3}$ Rothamsted Research, Harpenden, Hertfordshire, AL5 2QJ, UK; ${ }^{4}$ INRA Institut Jean-Pierre Bourgin, Station Géné- \\ tique et d'Amélioration des Plantes, F-78026 Versailles, France
}

Author for correspondence:

Anne-Marie Chèvre

Tel: $+33(0) 29923485131$

Email: chevre@rennes.inra.fr

Received: 28 August 2009

Accepted: 9 December 2009

New Phytologist (2010) 186: 102-112

doi: $10.1111 /$ j.1469-8137.2010.03182.x

Key words: Brassica napus (oilseed rape), homoeologous recombination, meiosis, polyploidy, synthetic hybrids.

\section{Summary}

- Polyploidy promotes the restructuring of merged genomes within initial generations of resynthesized Brassica napus, possibly caused by homoeologous recombination at meiosis. However, little is known about the impact of the first confrontation of two genomes at the first meiosis which could lead to genome exchanges in progeny. Here, we assessed the role of the first meiosis in the genome instability of synthetic $B$. napus.

- We used three different newly resynthesized B. napus plants and established meiotic pairing frequencies for the $A$ and $C$ genomes. We genotyped the three corresponding progenies in a cross to a natural $B$. napus on the two homoeologous $\mathrm{A} 1$ and $\mathrm{C} 1$ chromosomes. Pairing at meiosis in a set of progenies with various rearrangements was scored.

- Here, we confirmed that the very first meiosis of resynthesized plants of $B$. napus acts as a genome blender, with many of the meiotic-driven genetic changes transmitted to the progenies, in proportions that depend significantly on the cytoplasm background inherited from the progenitors.

- We conclude that the first meiosis generates rearrangements on both genomes and promotes subsequent restructuring in further generations. Our study advances the knowledge on the timing of genetic changes and the mechanisms that may bias their transmission.

\section{Introduction}

Compelling evidence has accumulated for ancient and/or recent genome-wide polyploidy among angiosperms (Doyle et al., 2008; Soltis et al., 2009; Van de Peer et al., 2009). Studies of natural allopolyploids (i.e. hybrids possessing two or more different sets of chromosomes) have revealed their divergence from the simple addition of progenitor genomes. Numerous genetic and epigenetic mechanisms have been shown to generate the wide range of structural and functional genome modifications associated with merged genomes arising from polyploidy events (Comai, 2005; Chen \& Ni, 2006; Leitch \& Leitch, 2008).

Some newly formed or resynthesized allopolyploids seem to be quiescent with few changes in genome organization, such as cotton and Spartina (Liu et al., 2001; Baumel et al., 2002), whereas most others are extensively remodelled within a few generations. Pairing between nonhomologous chromosomes at meiosis is thought to be responsible for many genomic restructuring events, such as homoeologous translocations and aneuploidy, as chiasmatic pairs between homoeologous chromosomes and univalent formation lead to abnormal genetic composition in gametes (Pikaard, 2001; Leitch \& Leitch, 2008).

Resynthesized Brassica provides a very good example of meiosis-driven genome reshuffling in allopolyploids. The seminal work of Song et al. (1995) on resynthesized Brassica species was among the first to show that major genomic changes occur as a result of meiotic irregularities. Brassica napus, an amphidiploid polyploid originating from different spontaneous crosses between $B$ r rapa $(n=10)$ and $B$. oleracea $(n=9)$ (Song \& Osborn, 1992), has since become one of the most widely used models to address this issue further (Osborn et al., 2003; Pires et al., 2004; Udall et al., 
2005; Albertin et al., 2006; Lukens et al., 2006; Gaeta et al., 2007). However the timing of genetic changes remains unclear. For instance, Lukens et al. (2006) observed few rearrangements in the $\mathrm{S} 0$ generation of resynthesized $B$. napus polyploids, but advocated that undetected rearrangements after S0 meiosis (Parkin et al., 1995; Udall et al., 2005) may become visible in further generations. Song et al. (1995) confirmed that genomic restructuring was frequently observed at the fourth generation of selfing. By contrast, Gaeta et al. (2007) detected this phenomenon in some progeny plants of selfed pollinated So individuals, rearrangements becoming homozygous in the bulking process at the third generation of selfing for most collinear chromosomes, and at the fifth generation for almost all chromosomal pairs. In this latter study, distortion in the transmission of rearrangement was detected, with the $\mathrm{A}$ genome being more frequently retained than the $\mathrm{C}$ genome (Gaeta et al., 2007). All these observations do not necessarily indicate that genome merging in S0 plants creates a burst of rearrangements at the first meiosis, which could then freely segregate and become homozygous in the latter generations. Indeed, Udall et al. (2005) observed that de novo chromosomal rearrangements occurred frequently in the vicinity of pre-existing translocations, which may suggest that only a few rearrangements produced during the meiosis of S0 plants give rise to an increasing number of genome changes in the following generations. Additional complexity comes from the fact that resynthesized B. napus plants produced with various progenitors display different levels of meiotic irregularities (univalent and multivalent formation), suggesting that the extent of homoeologous recombination is influenced by the progenitor genotype and/or their combination (Prakash \& Hinata, 1980; Attia \& Robbelen, 1986).

In order to obtain knowledge on the timing of genetic changes that occurred among $B$. napus synthetics, we investigated the role of the first meiosis in generating rearrangements between homoeologous chromosomes, and determined the importance of selection for viable gametic configurations.

We focused our study on the homoeologous chromosomes A1 and C1, which are the most collinear within the haplotype, and the most frequently rearranged pair in synthetic B. napus (Gaeta et al., 2007). To take into account the effect of the genome progenitor on the nature and frequency of rearrangements, three different S0 synthetic amphidiploids were used. To determine the precise transmission frequencies associated with the duplication and deletion of genetic regions from $\mathrm{A} 1$ and $\mathrm{C} 1$, we derived large populations from crosses between the three synthetic amphidiploids and a natural $B$. napus.

We established, through molecular analysis and cytological observations, that the first meiosis generates the genetic changes observed in the progeny. This study also sheds light on the timing of rearrangements, their consequences and the possible mechanisms responsible for the stabilization of $B$. napus synthetics.

\section{Materials and Methods}

\section{Production of synthetic SO material and $\mathrm{F}_{1}$ crosses with} a natural $B$. napus

Three synthetic lines were produced by A-M. Chèvre et al. (unpublished) by crossing B. rapa L. and B. oleracea L., plants belonging to different cultigroups. Parental plants were the selfed progeny of a $B$. rapa plant ' $\mathrm{C} 1.3$ ' belonging to a fodder variety named 'chicon' var. rapifera and three homozygous doubled haploid lines: 'Z1', a B. rapa var. trilocularis oilseed variety (provided by K. C. Falk, Agriculture and Agri-Food Canada, Ottawa, ON, available on request), 'HDEM', a $B$. oleracea var. botrytis italica, and 'RC34', a B. oleracea var. alboglabra. Interspecific hybridization between HDEM (maternal parent) and Z1 led to a haploid synthetic called BoEMZ (B. oleracea as maternal parent, prefixed Bo). Reciprocal hybridization between RC and $\mathrm{C} 1.3$ led to the haploid synthetics BoRCC (B. oleracea as maternal parent) and $\operatorname{BrCRC}(B$. rapa as maternal parent, prefixed $\mathrm{Br}$ ). The corresponding doubled hybrids (BoEMZ38, BoRCC38 and BrCRC38, respectively) were obtained after colchicine treatment, as described by Chevre et al. (1989). Three populations of 87, 91 and 92 plants were obtained by crossing the three S0 synthetic plants as maternal parent to a natural B. napus cv. Darmor (an oilseed rape winter type line) as male parent, and were called BoEMZ38 $\times$ Darmor, BoRCC38 $\times$ Darmor and BrCRC38 × Darmor, respectively.

\section{Meiotic behaviour and chromosome counting of a panel of $F_{1}$ plants}

Floral buds were fixed in Carnoy's solution (ethanol-chloroform-acetic acid, $6: 3: 1$ ) for $24 \mathrm{~h}$ and stored in 50\% ethanol. Observations on pollen mother cells (PMCs) were performed on anthers squashed and stained in a drop of $1 \%$ acetocarmine solution; 18-43 PMCs per plant were observed at metaphase I to establish the meiotic behaviour of a panel of individuals from BoRCC $\times$ Darmor or BrCRC $\times$ Darmor populations.

Fluorescence in situ hybridization (FISH) to detect $\mathrm{A}-\mathrm{C}$ pairs at meiosis and translocation in $\mathrm{SO} \times$ Darmor progenies

Bacterial artificial chromosome (BAC) FISH analyses were carried out on meiotic chromosomes for synthetic $B$. napus BrCRC38, BoRCC38 and BoEMZ38 at metaphase I in PMCs. We used a repeated sequence within a BAC (BoB014O06 from B. oleracea) as a probe for the $\mathrm{C}$ genome 
sequences (Alix et al., 2008). This 'genomic in situ hybridization (GISH)-like' BAC labelling hybridized specifically to all $\mathrm{C}$ genome chromosomes in previous $B$. napus FISH experiments (Leflon et al., 2006; Nicolas et al., 2007, 2009). Briefly, BAC clone BoB $14 \mathrm{O} 06$ was provided by Warwick Horticultural Research International (Wellesbourne, Warwick, UK) (Howell et al., 2002), labelled by random priming with biotin-14-deoxyuridine triphosphate according to the manufacturer's protocol (Invitrogen, San Diego, CA, USA; Life Technologies) and used as a probe at 100 ng per slide. Fluorescence images were captured using a CoolSnap HQ camera (Photometrics, Tucson, AZ, USA) on an Axioplan 2 microscope (Zeiss, Oberkochen, Germany) and analysed using MetaVue (Universal Imaging, Downington, PA, USA).

\section{Mapping translocation in $\mathrm{F}_{1}$ plants on $\mathrm{A} 1$ and $\mathrm{C} 1$ chromosomes}

DNA extraction was performed as described by Lombard \& Delourme (2001). In order to establish a genetic mapping position for all A1 and C1 markers used (some loci that mapped to one homoeologue were unable to be mapped on the other because of a lack of polymorphism in all our populations), we integrated the $\mathrm{A} 1$ and $\mathrm{C} 1$ linkage groups from the progenitor lines $(\mathrm{C} 1.3 \times \mathrm{Z} 1 \mathrm{~F} 2$ and $\mathrm{C} 10 \times \mathrm{HDEM}$ F2 populations, respectively) with those from the B. napus DH Darmor-bzh $\times$ Yudal population (Lombard \& Delourme, 2001; Rocherieux et al., 2004; Delourme et al., 2006) using Biomercator (Arcade et al., 2004). The two linkage groups $A 1$ and $C 1$ are shown for each population (see Supporting Information Fig. S1). All microsatellite markers are publicly available and originated either from the Biotechnology and Biological Sciences Research Council, Swindon, Wiltshire, UK (prefixed with $\mathrm{Na}$, Ol or Ra; Lowe et al., 2004), CELERA Company, Alameda, California, USA (prefixed with CB, BRAS or MR; Piquemal et al. (2005)) or Agriculture and Agri-Food Canada (prefixed with $s \mathrm{~N}$ ). Markers prefixed with the letters $C Z$ have been developed from coding sequences of Arabidopsis and Brassica (H. Belcram \& B. Chalhoub, unpublished; primer sequences are available on request to Genoplante). PCR assays were conducted essentially as described in Delourme et al. (2006). Forward primers were tailed with an M13 extension to be revealed by fluorescent technology (Schuelke, 2000). PCR products were analysed on a 16-capillary ABIPrism 3130xl, as described by Esselink et al. (2004). We scored the frequency of rearrangements in gametes by surveying the disappearance of parental bands from synthetic $B$. napus (i.e. A1 loci from B. rapa; C1 loci from B. oleracea). To consider small- and large-scale rearrangements at our locus density, one marker loss per chromosome was considered to represent a rearrangement.
Determination of the allele copy number based on the allele dosage on a fluorescent capillary genetic analyser in an SO synthetic crossed to a natural B. napus

Fluorescent PCR analysis was used to determine the allele copy number of $\mathrm{A} 1$ and $\mathrm{C} 1$ chromosomes from the synthetic parents BoEMZ38, BoRCC38 and BrCRC38 in the crosses BoEMZ38 $\times$ Darmor, BoRCC $38 \times$ Darmor and BrCRC38 $\times$ Darmor. Data collection and statistical analysis were performed as described in Nicolas et al. (2007). Briefly, the peak area of the A1 or C1 marker from the synthetic plant was compared with the peak area of the recurrent parent in S0 $\times$ Darmor progenies. Linear relationships could be visually identified for six loci (five markers), allowing the distinction of three lines, one with the loss of the synthetic allele, one with a single dose of the synthetic allele and the last with a double dose of the synthetic allele. To adjust the quality of the data, individuals with a peak area lower than 600 for either the synthetic peak or the recurrent parent peak were considered as missing values. We performed statistical analysis according to Nicolas et al. (2007) and repeated the molecular assay for four loci that confirmed previous observations.

\section{Statistical analysis to detect the effect of rearrangements on $\mathrm{SO} \times$ Darmor meiosis}

Statistical analyses were performed on the number of univalents, multivalents and the frequency of cells with 19 bivalents, reflecting global pairing, aberrant pairing frequencies and normal gamete formation, respectively.

The effects of complete and partial nullisomy/trisomy, $\mathrm{A} 1$ or $\mathrm{C} 1$ rearrangements and $\mathrm{A} 1$ rearrangement sizes were tested for all the variables by ANOVA with the procedure GLM from SAS software (SAS Institute Inc., Cary, North Carolina, USA). The normality of the distribution of residuals was tested using Shapiro-Wilk's test. The homogeneity of variances was checked with Bartlett's test. We compared the classes of plants using the Student-Newman-Keuls' test $(\alpha=0.05)$.

\section{Results}

\section{Cytological evidence for $A-C$ recombination at first meiosis of synthetic $B$. napus}

We evaluated cytogenetically the extent to which A and C chromosomes pair at meiosis (metaphase I) to assess the effect of the first meiosis on the genesis of genetic changes. Metaphase I observations in PMCs for the three S0 plants BoEMZ38, BoRCC38 and BrCRC38 showed between $37.5 \%$ and $60 \%$ of cells with $10 \mathrm{AA}$ bivalents and nine CC bivalents, whereas $30-47.5 \%$ of cells had at least one pair involving A-C chromosomes (Table 1, Fig. 1). 
New

Phytologist

Table 1 Frequencies of metaphase I in pollen mother cells (PMCs) involving A and C pairing configuration at first meiosis of euploid S0 synthetic Brassica napus

\begin{tabular}{|c|c|c|c|c|c|c|c|}
\hline $\begin{array}{l}\text { Synthetic SO } \\
\text { plant }\end{array}$ & $\begin{array}{l}\text { No. obs. } \\
\text { cells }\end{array}$ & $\begin{array}{l}10 \mathrm{~A}-\mathrm{A}+9 \\
\mathrm{C}-\mathrm{C} \text { bivalents }(\%)\end{array}$ & $\begin{array}{l}\text { Cells with } \\
\text { univalent } A \\
\text { and/or C (\%) }\end{array}$ & $\begin{array}{l}\text { Cells with } \\
\text { one A-C } \\
\text { bivalent (\%) }\end{array}$ & $\begin{array}{l}\text { Cells with } \\
\text { two or more } \\
\text { A-C bivalents (\%) }\end{array}$ & $\begin{array}{l}\text { Multivalent } \\
\text { involv. A-C } \\
\text { chr. (\%) }\end{array}$ & $\begin{array}{l}\text { Cells with } \\
\text { A-C } \\
\text { pairs (\%) }\end{array}$ \\
\hline BoEMZ38 & 40 & 40 & 33 & 7.50 & 35 & 10 & 47.50 \\
\hline BoRCC38 & 20 & 60 & 15 & 5 & 15 & 10 & 30 \\
\hline BrCRC38 & 16 & 37.50 & 37.50 & 6.25 & 18.75 & 12.50 & 31.25 \\
\hline
\end{tabular}

Fig. 1 A-C bivalents and multivalents at metaphase I of first meiosis of euploid SO synthetic Brassica napus. Unlabelled chromosomes (A genome) are in 4',6-diamidino-2phenylindole (blue, left) and Bob14006 (GISH-like signal on the $C$ genome) is stained in red by avidin-Texas red antibody (right). (a) EMZ.38 plant with two A-C bivalents (white arrows); (b) EMZ.38 plant with three A-C bivalents (white arrows) plus two univalents, one $A$ and one $C$ (white stars); (c) CRC.38 plant with one quadrivalent (AACC) (white arrow) plus two A univalents (white stars).
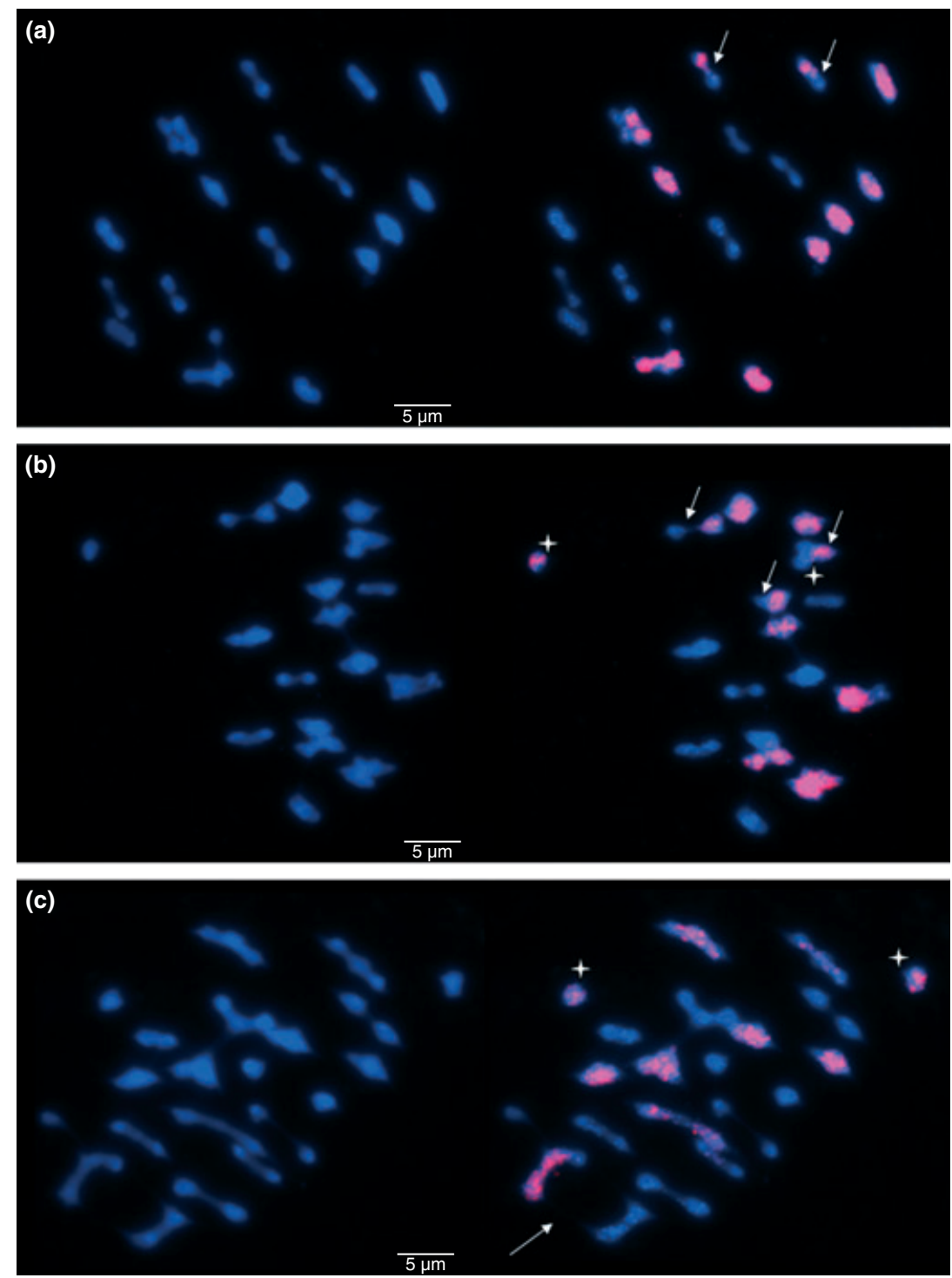

Owing to the frequent occurrence of $\mathrm{A}-\mathrm{C}$ bivalents and/or multivalents, gametes with unbalanced chromosomal composition and/or carrying chromosomal rearrangements were both expected, unless they were eliminated by selection. Molecular markers were used to decipher which of the two situations occurred for A1 and C1.
Detection of trisomics/monosomics in the progeny of SO synthetic $B$. napus

We assayed the parental marker transmission in the three populations produced by crossing the three S0 synthetic allotetraploids with the same natural $B$. napus genotype. Eight markers located on $\mathrm{A} 1$ and 11 markers located on $\mathrm{C} 1$ 


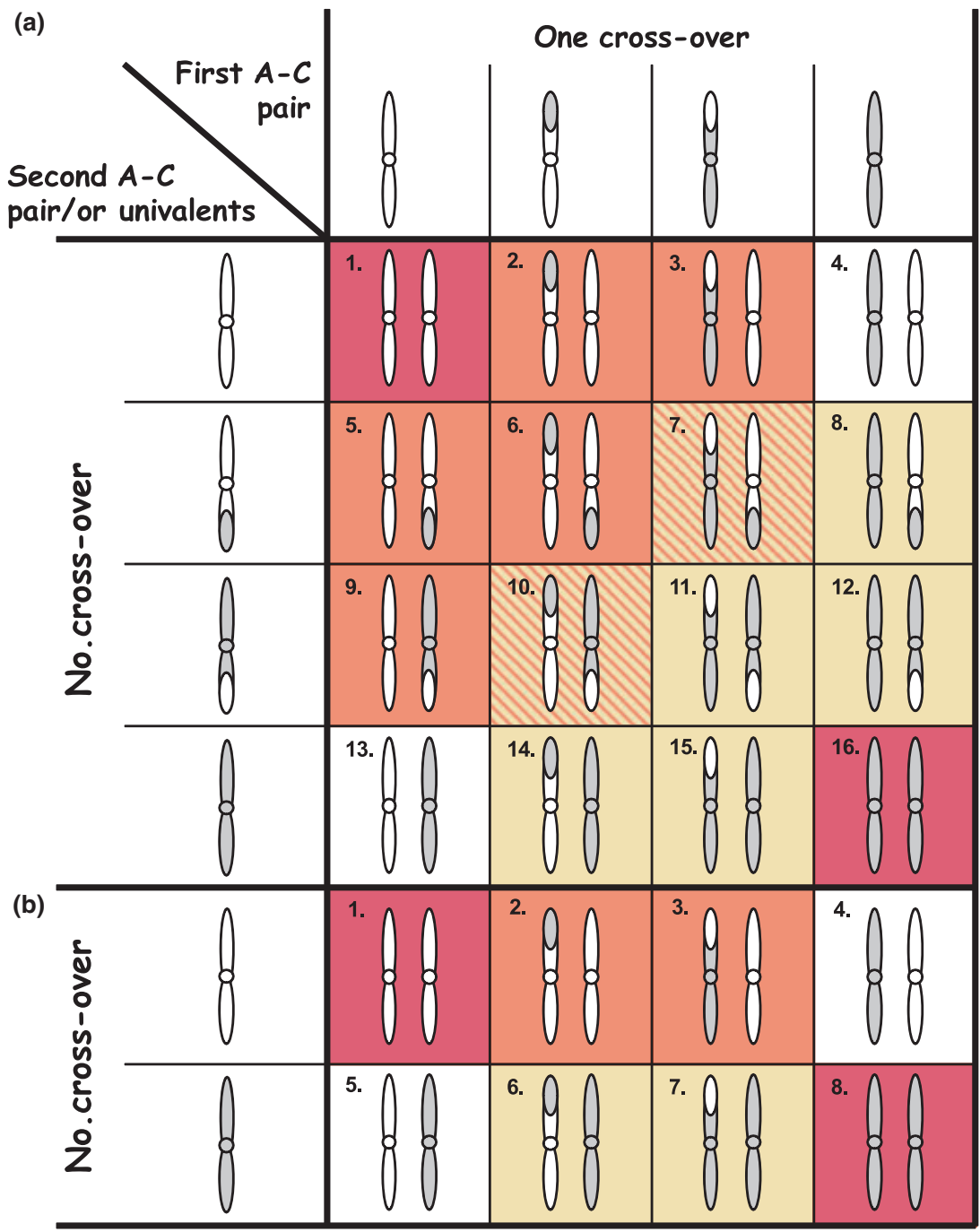

Fig. 2 Expected genomic structure in gametes $(n=19)$ resulting from cross-over in the presence of one or two A1-C1 bivalents at first meiosis of synthetic Brassica napus. Chromosome in white represents the $\mathrm{A} 1$ chromosome, and chromosome in grey represents the $\mathrm{C} 1$ chromosome. Translocations resulting from homoeologous pairing are represented by ellipses in the respective colour to their chromosomal origin. Two cases of meiosis are represented, in which one A1-C1 pair is formed (one cross-over) and for the remaining $\mathrm{A} 1$ and $\mathrm{C} 1$ chromosomes: (a) a second $\mathrm{A} 1-\mathrm{C} 1$ pair occurs with a cross-over at a different position, or (b) two univalents segregate in the gametes. Noncoloured cells [cells 4 and 13 for (a), cells 4 and 5 for (b)] represent parental-type gametes without translocations and chromosome loss. Cells in dark red [cells 1 and 16 for (a), cells 1 and 8 for (b)] represent gametes with two copies of the homoeologous chromosome and the loss of the reciprocal one. Orange cells [cells 2, 3, 5, 6 and 9 for (a), cells 2 and 3 for (b)] represent gametes carrying $\mathrm{C} 1$ region loss,

whereas light-brown cells [cells 8, 11, 12, 14 and 15 for (a), cells 6 and 7 for (b)] represent gametes carrying $A 1$ region loss. Striped orange and light brown cells [cells 10 and 7 for (a)] represent gametes carrying both $\mathrm{A} 1$ and C1 marker losses.
(Fig. S1) were used to genotype these populations, with six $\mathrm{A} 1$ and four $\mathrm{C} 1$ markers being common to all populations. These markers allowed the integration of at least $80 \%$ of the $\mathrm{A} 1$ and $\mathrm{C} 1$ linkage groups.

Over the 270 plants from the three populations, 5.2\% of plants $(4.6 \%, 5.4 \%$ and $5.5 \%$ for BoEMZ38 $\times \mathrm{D}$, BoRCC38 $\times \mathrm{D}$ and BrCRC38 $\times \mathrm{D}$, respectively) lacked all the markers from one parental linkage group (either A1 or C1), whereas markers of the homoeologous linkage group were present. Cytological counting of chromosomes on these plants indicated that they all had 38 chromosomes. Among the 15 plants that could be analysed for the dosage of synthetic alleles, six plants (out of seven) showed the concurrent loss of markers from A1 and the duplication of markers from $\mathrm{C} 1$, whereas eight plants (out of eight) showed the concurrent loss of markers from $\mathrm{C} 1$ and duplication of markers from A1. Cytological counting indicated that all 14 plants had 38 chromosomes per PMC, which suggests that they were trisomic for one chromosome and monosomic for its homoeologue (carrying an A1 and a $\mathrm{C} 1$ from the natural B. napus cv. Darmor paternal gamete). Fig. 2 provides a model showing that gametes with a normal chromosome number, i.e. 19 chromosomes, can carry two copies of A1 (with no C1; Fig. 2(a,b), cell 1) or two copies of C1 (with no A1; Fig. 2(a), cell 16; Fig. 2(b), cell 8) when one or two A1-C1 bivalents are formed, therefore called nullisomic/disomic gametes.

As expected from the meiotic behaviour of S0 resynthesized B. napus, monosomics/trisomics for A1 and C1 were detected in the progeny of these synthetics. These plants were discarded from further analysis to avoid biasing the deletion frequency per locus. Therefore, populations were reduced to 82,87 and 86 individuals for BoEMZ38 $\times$ Darmor, BoRCC38 $\times$ Darmor and BrCRC $38 \times$ Darmor, respectively (255 plants). 
New
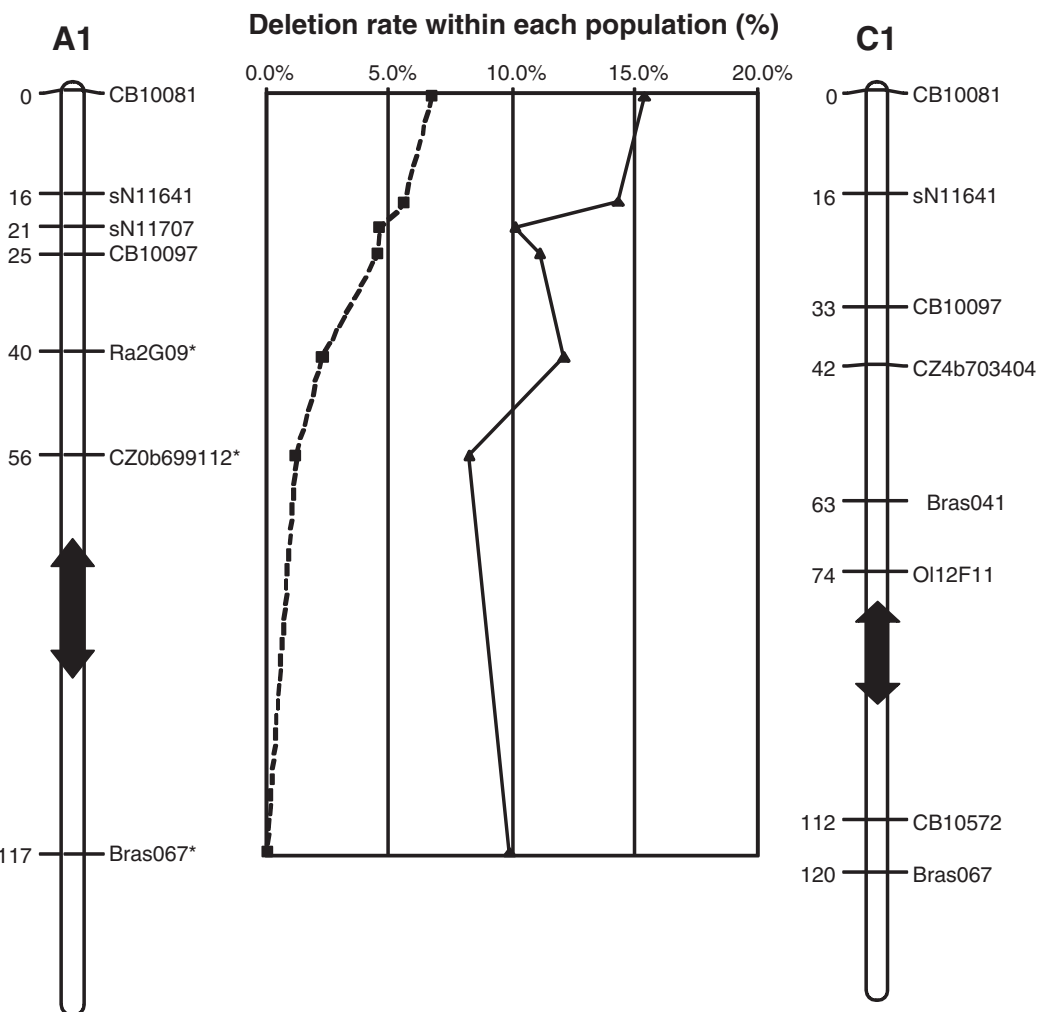

Deletion rate within each population (\%)

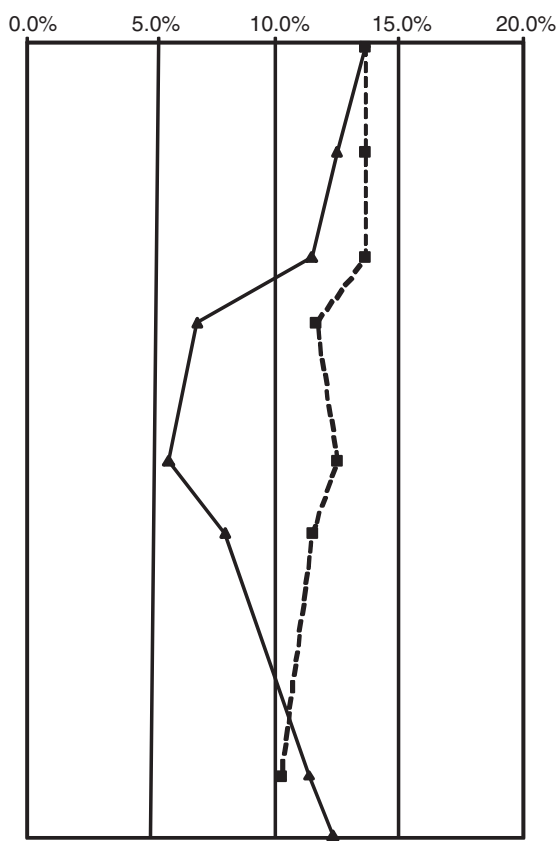

Fig. 3 Observed deletion rate of alleles from A1 or C1 linkage groups following first meiosis of two S0 synthetic Brassica napus in the $B$. rapa cytoplasm population BrCRC38 $\times$ Darmor (squares and broken line) or the B. oleracea cytoplasm population BoRCC38 $\times$ Darmor (triangles and full line). Significant differences between the deletion rates of the two populations are indicated with an asterisk. Black arrows represent the presumed position of centromeres according to Pouilly et al. (2008). Cumulated genetic distances (Kosambi) are indicated on the left side of the linkage groups in centimorgans.

\section{Detection of chromosomal rearrangements in the progeny of SO synthetic $B$. napus}

We genotyped the progeny of synthetic $B$. napus to detect chromosomal rearrangements, resulting in the loss of some but not all markers of a given linkage group. Plants carrying rearrangements on $\mathrm{A} 1$ and/or C1 represented $40.4 \%$ of the 255 plants observed. Plants with rearrangements on both $\mathrm{A} 1$ and $\mathrm{C} 1$ chromosomes accounted for $10.6 \%$. None of the 255 plants surveyed displayed a concurrent loss of both homoeologous loci on $\mathrm{A} 1$ and $\mathrm{C} 1$ chromosomes. Considering 510 total $\mathrm{A} 1$ and $\mathrm{C} 1$ chromosomes in the 255 plants, $74.3 \%$ were not rearranged, $19.4 \%$ had a single breakpoint and $6.3 \%$ had multiple breakpoints. Single breakpoints along the chromatid explain the difference between the frequencies of marker losses along A1 and C1 chromosomes, with distal markers tending to be more frequently lost than pericentromeric ones (see Fig. 3 for an example on BoRCC $38 \times$ Darmor and BrCRC38 $\times$ Darmor populations).

To estimate the rate of marker loss as a result of crossovers between $\mathrm{A} 1$ and $\mathrm{C} 1$ at meiosis, we determined whether any loss of marker on A1 was associated with the duplication of markers from the $\mathrm{C} 1$ homoeologous region (and vice versa). Concurrent loss and duplication were examined for six homoeologous loci (see Materials and Methods). Among 24 loss events on A1, where duplicated regions could be scored, 18 (75\%) were associated with duplication of the $\mathrm{C} 1$ allele, whereas, for 48 loss events on C1, where duplicated regions could be detected, 44 $(91.7 \%)$ individuals showed duplication of A1. Among the 255 plants studied, seven $(2.7 \%)$ presented a double dose of A1 without any marker loss on both chromosomes, whereas seven others (2.7\%) showed the reciprocal situation with a double dose of $\mathrm{C} 1$. One plant $(0.4 \%)$ had both $\mathrm{A} 1$ and $\mathrm{C} 1$ doubled without any deletions.

Our marker data confirmed that the first meiosis of resynthesized $B$. napus generates extensive genetic changes (plants with unbalanced chromosome composition, chromosomal rearrangements) that are transmitted to the progenies. This indicated that all genetic changes were not discarded by selection. We then examined whether differences could be detected among the three populations. 
Table 2 Percentages of plants carrying rearrangements on A 1 and/or C1 chromosomes in the progeny of each S0 synthetic crossed to a natural Brassica napus

\begin{tabular}{llll}
\hline Population & $\begin{array}{l}\text { BoEMZ38 } \times \\
\text { Darmor }(\%)\end{array}$ & $\begin{array}{l}\text { BoRCC38 } \times \\
\text { Darmor }(\%)\end{array}$ & $\begin{array}{l}\text { BrCRC38 } \times \\
\text { Darmor }(\%)\end{array}$ \\
\hline A1 total & 30.50 & 26.40 & 7 \\
C1 total & 37.80 & 26.40 & 25.60 \\
Total & 50.00 & 41.40 & 30.20 \\
\hline
\end{tabular}

Populations were composed of 82,87 and 86 individuals for BoEMZ38 $\times$ Darmor, BoRCC38 $\times$ Darmor and BrCRC38 $\times$ Darmor, respectively.

Some rearrangements are differentially counterselected in a population-dependent and chromosomedependent manner

We compared the frequencies of rearrangements between homoeologous chromosomes, the distribution of rearrangements along the chromosomes and the type of rearrangements (using breakpoint number per plant and chromosome as an estimator) among the three populations.

Significant differences were observed in the progenies of the reciprocal synthetics (BrCRC38 vs BoRCC38) that shared a common nuclear genetic background but different maternally donated cytoplasms. BrCRC38 $\times$ Darmor showed an excess of plants without rearrangement $(69.8 \%$ vs $58.6 \%$, respectively, $P<0.01, \chi^{2}$ test) and a lower frequency of plants carrying A1 marker loss (7\% vs $26.4 \%$, respectively, $P<0.05, \chi^{2}$ test) than BoRCC38 $\times$ Darmor (Table 2). Multiple breakpoints were less frequent on $A 1$ in the BrCRC38 $\times$ Darmor population compared with BoRCC38 $\times$ Darmor $\left(P<0.05, \chi^{2}\right.$ test between the distribution for the two populations). By contrast, no difference was found between $\mathrm{C} 1$ marker loss frequencies in the progenies of BoRCC38 and BrCRC38.

Conversely, populations BoRCC38 $\times$ Darmor and BoEMZ38 $\times$ Darmor with a $B$. oleracea cytoplasm were not significantly different for: (1) the frequency of plants carrying marker losses; (2) the frequency of losses along A1 and C1 chromosomes; (3) the breakpoint number per chromosome (Table 3). These data showed that the genetic backgrounds on $B$. oleracea cytoplasm did not influence the frequency of rearrangements.
Our results suggest that the frequency and size of chromosomal translocations are biased in a population-dependent manner in the progenies of SO B. napus synthetics. We therefore investigated whether these rearrangements would also affect the regularity of meiosis in offspring carrying genetic changes.

Rearrangements and their 'size' condition the normal meiotic behaviour of SO $\times$ Darmor progenies

We established the meiotic behaviour for 17 plants from BoRCC3 $8 \times$ Darmor and BrCRC38 $\times$ Darmor populations (Table 4), selected on the basis of the size of their rearrangements, ranging from intact $\mathrm{A} 1$ and $\mathrm{C} 1$ chromosomes to complete exchange of a chromosome. To represent the meiotic behaviour with synthetic variables, we scored univalent and multivalent frequencies to reflect the pairing ability between chromosomes, and the frequency of cells with 19 bivalents to reflect the formation of normal gametes.

We first tested the impact of complete and partial monosomic/trisomic plants on the meiotic behaviour, and found that plants monosomic for $\mathrm{A} 1$ and trisomic for $\mathrm{C} 1$ showed significantly more univalents and a lower ratio of cells with 19 bivalents $(P<0.01$, ANOVA test $)$ than controls that had a balanced (one A1 and one C1 inherited from the resynthesized parent) composition. By contrast, plants with the reciprocal genetic structure (complete or partial monosomic plants for $\mathrm{C} 1$ and trisomic for A1) were not significantly different from controls.

We then tested the impact of chromosomal rearrangements on $\mathrm{A} 1$ or $\mathrm{C} 1$ on the regularity of meiosis. Plants rearranged for $\mathrm{A} 1$ or $\mathrm{C} 1$ showed significantly more univalents than nonrearranged plants $(P<0.05$, ANOVA test $)$, but the accumulation of both $\mathrm{A} 1$ and $\mathrm{C} 1$ rearrangements did not induce more univalents than expected.

To assess the effect of rearrangement size further, we focused on the A1 chromosome and contrasted plants without rearrangements (BoRCC38 $\times$ Darmor024, BrCRC38 $\times$ Darmor068 and BrCRC38 $\times$ Darmor004 in Table 4), plants with subtelomeric rearrangements (BrCRC $38 \times$ Darmor027 and BoRCC38 $\times$ Darmor077 in Table 4) and plants with a half-arm loss (BoRCC $38 \times$ Darmor073 and BoRCC38 $\times$ Darmor011 in Table 4). The latter plants

Table 3 Repartition of plants according to the number of breakpoints per rebuilt linkage group (LG) A1 and C1 in the progeny of a threesynthetic-SO Brassica napus crossed with cv. Darmor

\begin{tabular}{|c|c|c|c|c|c|c|c|}
\hline Breakpoints per LG & & 0 & 1 & 2 & 3 & 4 & Total LG \\
\hline BoEMZ38 × Darmor & A1 & 58 & 13 & 10 & 0 & 1 & 82 \\
\hline B. oleracea cytoplasm & C1 & 51 & 22 & 8 & 1 & 0 & 82 \\
\hline BoRCC38 × Darmor & A1 & 62 & 19 & 4 & 2 & 0 & 87 \\
\hline B. oleracea cytoplasm & C1 & 64 & 18 & 4 & 0 & 1 & 87 \\
\hline BrCRC38 × Darmor & A1 & 80 & 6 & 0 & 0 & 0 & 86 \\
\hline B. rapa cytoplasm & C1 & 64 & 21 & 1 & 0 & 0 & 86 \\
\hline
\end{tabular}


New

Phytologist

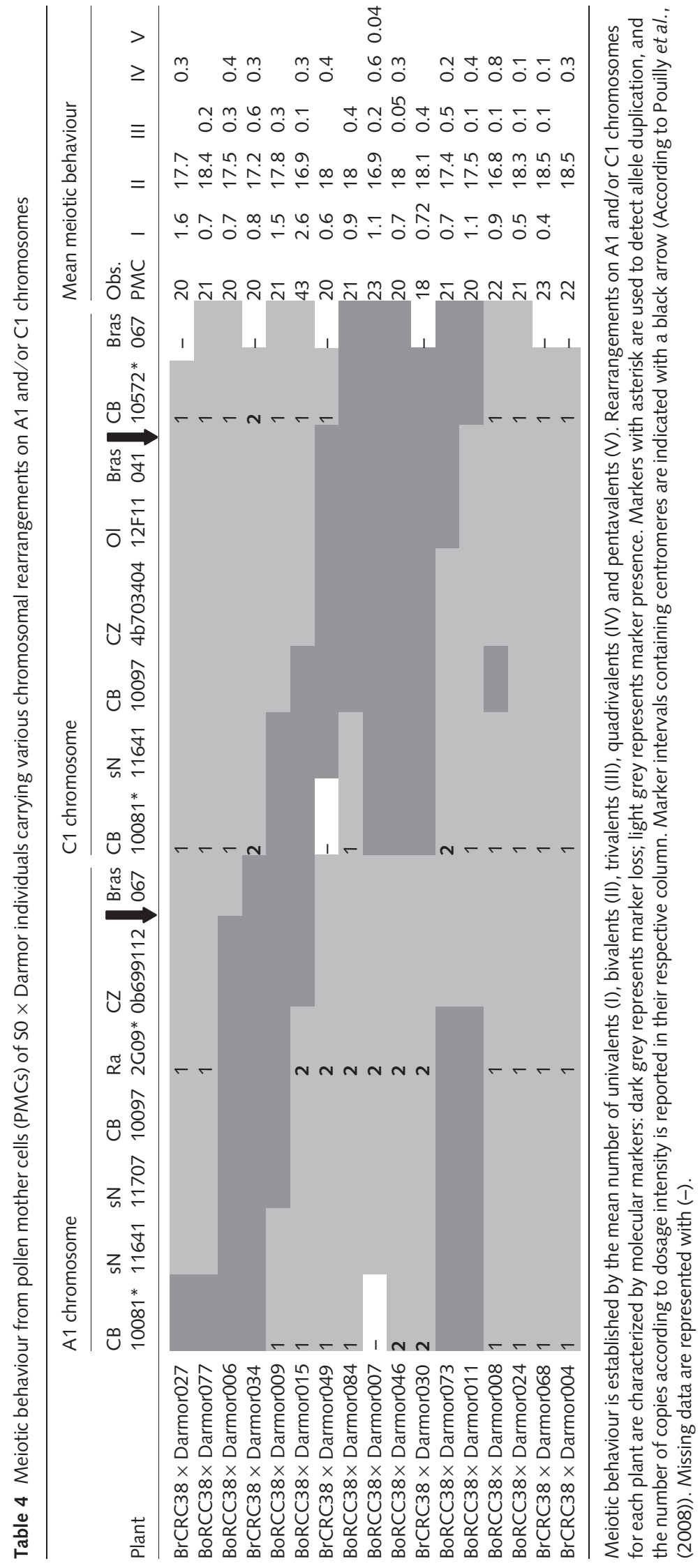




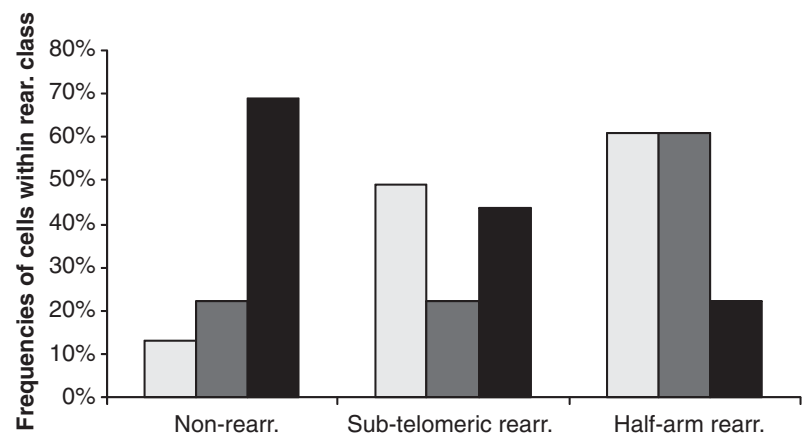

Fig. 4 Effect of genetic size of rearrangement on meiotic behaviour in S0 $\times$ Darmor progenies. Percentage of cells with univalent(s) (light grey), multivalent(s) (dark grey) and 19 bivalents (black) on plants without rearrangements (percentage based on three plants), with subtelomeric rearrangements (percentage based on two plants) and half-arm rearrangement (percentage based on two plants).

showed more univalents and multivalents than nonrearranged plants (Fig. 4), and therefore had fewer cells carrying 19 bivalents $(P<0.05$, ANOVA test). By contrast, plants with subtelomeric rearrangements had intermediate frequencies, but were not significantly different from both classes of plants.

All these results suggest that viable genetic changes produced during the meiosis of S0 synthetics may have an effect on the occurrence of new genetic changes in later generations.

\section{Discussion}

Meiosis drives extensive genetic changes that are transmitted to the progenies of SO B. napus synthetics

Our results showed that the meiosis of resynthesized $B$. napus acts as a genome blender.

Cytological observations first showed that recombination frequently occurred between homoeologous chromosomes. For the three synthetics analysed, metaphase I A-C bivalents and multivalents were present in 30-47.5\% of PMCs, and up to $10 \%$ of total cells had more than three A-C chromosome associations. These findings extend the observations of multivalents by Attia \& Robbelen (1986) and Prakash \& Hinata (1980), thanks to the use of BAC FISH with a 'GISH like' pattern that allows the discrimination of $\mathrm{A}$ and $\mathrm{C}$ chromosomes.

We then demonstrated that extensive genetic changes were transmitted to the progenies of resynthesized B. napus. Over all populations, $5.2 \%$ of plants had an abnormal chromosomal composition [whilst carrying 38 chromosomes; see Lim et al. (2008) for a similar example in natural Tragopogon populations] and $40.4 \%$ of plants carried rearrangements on $\mathrm{A} 1$ and/or $\mathrm{C} 1$ chromosomes, at a higher rate compared with Udall et al. (2005). These genetic changes were expected as a result of chromosome mis-segregation and/or cross-overs between homoeologous chromosomes during the meiosis of S0 synthetics (see Pikaard, 2001; Leitch \& Leitch, 2008).

Finally, our molecular data confirmed that most chromosomal rearrangements $(81.8 \%$ on $\mathrm{A} 1$ and $94.4 \%$ on $\mathrm{C} 1$ ) were likely to be the products of cross-overs between homoeologous A1 and $\mathrm{C} 1$ chromosomes, because they resulted in the concurrent loss and duplication of the homoeologous region (Gaeta et al., 2007; Nicolas et al., 2007). This rate is slightly higher than that obtained in the progenies of haploids from natural B. napus on A1-C1 (73\%; Nicolas et al., 2007, 2009). In addition, the occurrence of multiple breakpoints for $6.3 \%$ of the rearranged chromosomes suggests that more than one cross-over per homoeologous A1-C1 chromosome would occur at first meiosis, although part of these rearrangements could also originate from one single breakpoint on each pair of A-C bivalents (Fig. 2(a), cells 6 and 11). Our results are coherent with those from natural B. napus that confirm pre-existing $\mathrm{A} 1$ and $\mathrm{C} 1$ translocations in varieties and the ability of this homoeologous pair to recombine de novo (Udall et al., 2005), with a similar increase in deletion frequencies towards telomeres for haploids of natural B. napus (Nicolas et al., 2007).

According to our results, we would expect to detect rearrangements in the first selfing progeny of S0 synthetic $B$. napus, which have already been detected before the bulking process for a few loci and plants in previous work on $B$. napus synthetics with a $B$. oleracea cytoplasm (Gaeta et al., 2007). However, large selfing populations would be required to detect accurately such genetic changes, because similar rearrangements must be generated and transmitted through male and female meioses to the same S1. Several successive generations, up to third or fifth according to Song et al. (1995) and Gaeta et al. (2007), are usually required for a rearrangement to become homozygous in a selfed plant (Gaeta \& Pires, 2010).

\section{Different genome changes are observed in the progenies of reciprocal synthetics}

Although our results have indicated that many of the meiosis-driven genetic changes are transmitted, comparisons of the progenies of the reciprocal synthetics suggest that cytoplasm-dependent selective effects may also bias the transmission of some rearrangements.

Comparison of the BoRCC38 $\times$ Darmor and BrCRC $38 \times$ Darmor populations revealed a bias in favour of A1 chromosome retention in BrCRC $38 \times$ Darmor progeny, without any effect on the frequency of $\mathrm{C} 1$ rearrangements. As the two synthetic S0 parents (BoRCC38 and BrCRC38) shared the same nuclear genetic background and displayed almost the same proportion of cells showing $\mathrm{A}-\mathrm{C}$ chiasmatic associations (Table 1), we would hypothesize that some rearrangements resulting in the loss of DNA from A1 were counterselected and discarded from the progeny of SO synthetic 
BrCRC38 (gametic or zygotic selection) that carried a $B$. rapa cytoplasm. Presumably, the preferential retention of A1 DNA in the progeny of BrCRC38 could thus be the result of nucleocytoplasmic interactions that were not detected with the B. oleracea cytoplasm (Tsunewaki, 1993). It is noteworthy that Song et al. (1995) did not detect any significant cytoplasmic effect on the transmission of rearrangements between (advanced) reciprocal synthetic $B$. napus, but a significantly biased loss of B-genome restriction fragment length polymorphisms among F5 individuals derived from an initial AABB synthetic allopolyploid generated with the A cytoplasm. Our hypothesis thus needs to be evaluated by further studies.

By contrast with the comparison of BoRCC38 $\times$ Darmor and BrCRC38 $\times$ Darmor populations, we did not detect any difference in genetic changes on $\mathrm{A} 1-\mathrm{C} 1$ in the progenies of BoEMZ38 and BoRCC38 that both carried the $B$. oleracea cytoplasm (Table 3 ). At first sight, this result is in contrast with the difference in meiotic behaviour observed between the two plants, BoEMZ38 showing more cells with A-C associations than BoRCC38. However, this difference mostly originated from a larger number of cells showing two or more A-C bivalents in BoEMZ38 compared with BoRCC38 (Table 1), suggesting that another pair of homoeologues rather than $\mathrm{A} 1-\mathrm{C} 1$ may recombine in BoEMZ38 but not in BoRCC38. Notwithstanding the validity of this hypothesis, cytoplasm-specific and chromosome-specific differences in rearrangement frequency can be expected among the progenies of different S0 synthetics. Our results suggest that this would affect the regularity of meiosis in plants of the subsequent generations, especially if they carry rearrangements of different size.

\section{Chromosomal restructuring at the heterozygous stage from the first meiosis disturbs the meiosis of $\mathrm{SO} \times$ Darmor progenies}

We observed that the larger the rearrangements, the less regular the meiosis. This sounds like a truism at first sight, but specific comparisons provide considerably more information. Notably, we observed that the regularity of meiosis was more severely affected by the loss of chromosome A1 than the loss of C1. Although we do not know what is responsible for this difference, this result provides a potential explanation of why more frequent and larger $\mathrm{C}$ genomic regions (compared with A genomic regions) are lost in subsequent selfing generations (Gaeta et al., 2007). In addition, rearrangements were shown to hamper normal chromosome pairing and recombination during meiosis; thus, they are likely to increase the frequency of restructuring in subsequent generations, certainly much further than at the fifth generation of selfing (A-M. Chèvre, unpublished), and are responsible for the polyploid ratchet described by Gaeta et al. (see detailed explanations in Gaeta \& Pires, 2010). It is probable that the establishment of a genetic control of homoeologous pairing (described in B. napus by Jenczewski et al., 2003), as well as illegitimate recombination (Wang et al., 2009), contribute to stabilize the genomes after several successive generations and under purifying selection.

Our results indicate that, in $B$. napus synthetics, genome restructuring by homoeologous recombination at first meiosis is influenced by cytoplasmic interactions. This process is ongoing over generations, creating novel ratios of homoeologous regions that modulate the plant phenotype (see Pires et al., 2004 for flowering time), and should be addressed in terms of evolutionary consequences.

\section{Acknowledgements}

We thank Jean-Claude Letanneur for technical assistance. Joseph Jahier (INRA Rennes, France) is gratefully acknowledged for critical reading of the manuscript and Biogenouest ${ }^{\circledR}$ (Le Rheu, France) for genotyping facilities. Emmanuel Szadkowki was supported by a fellowship from the French Research Ministry (MENRT). This work was carried out with the financial support of the 'ANR - Agence Nationale de la Recherche - The French National Research Agency' under the 'Programme Biodiversité, project 'ANR-05BDIV-015, Effet de la polyploïdie sur la biodiversité et l'évolution du génome des plantes'.

\section{References}

Albertin W, Balliau T, Brabant P, Chevre AM, Eber F, Malosse C, Thiellement H. 2006. Numerous and rapid nonstochastic modifications of gene products in newly synthesized Brassica napus allotetraploids. Genetics 173: 1101-1113.

Alix K, Joets J, Ryder CD, Moore J, Barker GC, Bailey JP, King GJ, Heslop-Harrison JS. 2008. The CACTA transposon Bot1 played a major role in Brassica genome divergence and gene proliferation. Plant Journal 56: 1030-1044.

Arcade A, Labourdette A, Falque M, Mangin B, Chardon F, Charcosset A, Joets J. 2004. BioMercator: integrating genetic maps and QTL towards discovery of candidate genes. Bioinformatics 20: 2324-2326.

Attia T, Robbelen G. 1986. Meiotic pairing in haploids and amphidiploids of spontaneous versus synthetic origin in rape, Brassica napus L. Canadian Journal of Genetics and Cytology 28: 330-334.

Baumel A, Ainouche M, Kalendar R, Schulman AH. 2002. Retrotransposons and genomic stability in populations of the young allopolyploid species Spartina anglica CE Hubbard (Poaceae). Molecular Biology and Evolution 19: 1218-1227.

Chen ZJ, Ni ZF. 2006. Mechanisms of genomic rearrangements and gene expression changes in plant polyploids. Bioessays 28: 240-252.

Chevre AM, Eber F, Thomas G, Baron F. 1989. Cytological studies of tetraploid kale (Brassica oleracea L. ssp acephala) obtained from diploid lines after colchicine treatment. Agronomie 9: 521-525.

Comai L. 2005. The advantages and disadvantages of being polyploid. Nature Reviews Genetics 6: 836-846.

Delourme R, Falentin C, Huteau V, Clouet V, Horvais R, Gandon B, Specel S, Hanneton L, Dheu JE, Deschamps M et al. 2006. Genetic control of oil content in oilseed rape (Brassica napus L.). Theoretical and Applied Genetics 113: 1331-1345. 
Doyle JJ, Flagel LE, Paterson AH, Rapp RA, Soltis DE, Soltis PS, Wendel JF. 2008. Evolutionary genetics of genome merger and doubling in plants. Annual Review of Genetics 42: 443-461.

Esselink GD, Nybom H, Vosman B. 2004. Assignment of allelic configuration in polyploids using the MAC-PR (microsatellite DNA allele counting-peak ratios) method. Theoretical and Applied Genetics 109: 402-408.

Gaeta RT, Pires JC. 2010. Homoeologous recombination in allopolyploids: the polyploid ratchet. New Phytologist 186: 18-28.

Gaeta RT, Pires JC, Iniguez-Luy F, Leon E, Osborn TC. 2007. Genomic changes in resynthesized Brassica napus and their effect on gene expression and phenotype. Plant Cell 19: 3403-3417.

Howell EC, Barker GC, Jones GH, Kearsey MJ, King GJ, Kop EP, Ryder CD, Teakle GR, Vicente JG, Armstrong SJ. 2002. Integration of the cytogenetic and genetic linkage maps of Brassica oleracea. Genetics 161: $1225-1234$.

Jenczewski E, Eber F, Grimaud A, Huet S, Lucas MO, Monod H, Chevre AM. 2003. PrBn, a major gene controlling homeologous pairing in oilseed rape (Brassica napus) haploids. Genetics 164: 645-653.

Leflon M, Eber F, Letanneur JC, Chelysheva L, Coriton O, Huteau V, Ryder CD, Barker G, Jenczewski E, Chevre AM. 2006. Pairing and recombination at meiosis of Brassica rapa $(\mathrm{AA}) \times$ Brassica napus (AACC) hybrids. Theoretical and Applied Genetics 113: 1467-1480.

Leitch AR, Leitch IJ. 2008. Perspective - genomic plasticity and the diversity of polyploid plants. Science 320: 481-483.

Lim KY, Soltis DE, Soltis PS, Tate J, Matyasek R, Srubarova H, Kovarik A, Pires JC, Xiong Z, Leitch AR. 2008. Rapid chromosome evolution in recently formed polyploids in Tragopogon (Asteraceae). PLoS ONE3: e3353.

Liu B, Brubaker CL, Mergeai G, Cronn RC, Wendel JF. 2001. Polyploid formation in cotton is not accompanied by rapid genomic changes. Genome 44: 321-330.

Lombard V, Delourme R. 2001. A consensus linkage map for rapeseed (Brassica napus L.): construction and integration of three individual maps from DH populations. Theoretical and Applied Genetics 103: 491507.

Lowe AJ, Moule C, Trick M, Edwards KJ. 2004. Efficient large-scale development of microsatellites for marker and mapping applications in Brassica crop species. Theoretical and Applied Genetics 108: 1103-1112.

Lukens LN, Pires JC, Leon E, Vogelzang R, Oslach L, Osborn T. 2006. Patterns of sequence loss and cytosine methylation within a population of newly resynthesized Brassica napus allopolyploids. Plant Physiology 140: 336-348.

Nicolas SD, Leflon M, Monod H, Eber F, Coriton O, Huteau V, Chevre AM, Jenczewski E. 2009. Genetic regulation of meiotic cross-overs between related genomes in Brassica napus haploids and hybrids. Plant Cell 21: 373-385.

Nicolas SD, Le Mignon G, Eber F, Coriton O, Monod H, Clouet V, Huteau V, Lostanlen A, Delourme R, Chalhoub B et al. 2007. Homeologous recombination plays a major role in chromosome rearrangements that occur during meiosis of Brassica napus haploids. Genetics 175: 487503.

Osborn TC, Butrulle DV, Sharpe AG, Pickering KJ, Parkin IAP, Parker JS, Lydiate DJ. 2003. Detection and effects of a homeologous reciprocal transposition in Brassica napus. Genetics 165: 1569-1577.

Parkin IAP, Sharpe AG, Keith DJ, Lydiate DJ. 1995. Identification of the A and $\mathrm{C}$ genomes of amphidiploid Brassica napus (oilseed rape). Genome 38: $1122-1131$.
Pikaard CS. 2001. Genomic change and gene silencing in polyploids. Trends in Genetics 17: 675-677.

Piquemal J, Cinquin E, Couton F, Rondeau C, Seignoret E, Doucet I, Perret D, Villeger MJ, Vincourt P, Blanchard P. 2005. Construction of an oilseed rape (Brassica napus L.) genetic map with SSR markers. Theoretical and Applied Genetics 111: 1514-1523.

Pires JC, Zhao JW, Schranz ME, Leon EJ, Quijada PA, Lukens LN, Osborn TC. 2004. Flowering time divergence and genomic rearrangements in resynthesized Brassica polyploids (Brassicaceae). Biological Journal of the Linnean Society 82: 675-688.

Pouilly N, Delourme R, Alix K, Jenczewski E. 2008. Repetitive sequencederived markers tag centromeres and telomeres and provide insights into chromosome evolution in Brassica napus. Chromosome Research 16: 683700

Prakash S, Hinata K. 1980. Taxonomy cytogenetics and origin of crop brassicas as review. Opera Botanica 55: 1-57.

Rocherieux J, Glory P, Giboulot A, Boury S, Barbeyron G, Thomas G, Manzanares-Dauleux MJ. 2004. Isolate-specific and broad-spectrum QTLs are involved in the control of clubroot in Brassica oleracea. Theoretical and Applied Genetics 108: 1555-1563.

Schuelke M. 2000. An economic method for the fluorescent labeling of PCR fragments. Nature Biotechnology 18: 233-234.

Soltis DE, Albert VA, Leebens-Mack J, Bell CD, Paterson AH, Zheng C, Sankoff D, Pamphilis CWD, Wall PK, Soltis PS. 2009. Polyploidy and angiosperm diversification. American Journal of Botany 96: 336-348.

Song KM, Lu P, Tang KL, Osborn TC. 1995. Rapid genome change in synthetic polyploids of Brassica and its implications for polyploid evolution. Proceedings of the National Academy of Sciences, USA 92: 7719-7723.

Song K, Osborn TC. 1992. Polyphyletic origins of Brassica napus - new evidence based on organelle and nuclear RFLP analyses. Genome 35: 992-1001.

Tsunewaki K. 1993. Genome plasmon interactions in wheat. Japanese Journal of Genetics 68: 1-34.

Udall JA, Quijada PA, Osborn TC. 2005. Detection of chromosomal rearrangements derived from homeologous recombination in four mapping populations of Brassica napus L. Genetics 169: 967-979.

Van de Peer Y, Maere S, Meyer A. 2009. OPINION The evolutionary significance of ancient genome duplications. Nature Reviews Genetics 10: $725-732$.

Wang X, Tang H, Bowers JE, Paterson AH. 2009. Comparative inference of illegitimate recombination between rice and sorghum duplicated genes produced by polyploidization. Genome Research 19: 1026-1032.

\section{Supporting Information}

Additional supporting information may be found in the online version of this article.

Fig. S1 A1 and C1 linkage groups used for the three So $\times$ Darmor populations.

Please note: Wiley-Blackwell are not responsible for the content or functionality of any supporting information supplied by the authors. Any queries (other than missing material) should be directed to the New Phytologist Central Office. 